\title{
Combining Human and Machine Capabilities for Improved Accuracy and Speed in Visual Recognition Tasks
}

\author{
Amir Schur and Charles Tappert \\ Seidenberg School of CSIS, Pace University \\ 1 Martine Ave., White Plains, NY 10606, USA \\ amirschur@aol.com, ctappertepace.edu
}

\begin{abstract}
This study investigated methods of enhancing human computer interaction in applications of pattern recognition where higher accuracy is required than is currently achievable by automated systems, but where there is enough time for a limited amount of human interaction. On a flower identification task, methods were explored to improve the accuracy of humanonly and machine-only recognition by employing human-computer interaction. Human involvement in the feature extraction process, especially in color selection, was found to be most beneficial.
\end{abstract}

Keywords: Human-computer interaction, visual object recognition, pattern classification, feature extraction.

\section{Introduction}

In various areas of pattern recognition, particularly in visual recognition tasks, there is generally a trade-off between accuracy and speed. Having the task performed manually by an expert usually results in high accuracy but takes a significant amount of time to accomplish. Performing the task automatically by machine usually can be done rapidly but the accuracy level is often low. The visual recognition task areas can be facial recognition, hand-writing recognition, etc. While research can focus on how to create a tool to assist an expert or on how to create a better automated tool, another way of improving automated tools is by employing human assistance in tasks where the time required is still deemed acceptable. This study, therefore, investigates the employment of human assistance in the feature extraction process of an automated visual pattern recognition task.

This study uses a java-based software tool called IVS (Interactive Visual System) which was developed from the CAVIAR model in which humans and machines interact to identify flowers [1]. IVS can operate on handheld devices or a code simulator run on laptop/desktop machines (Figures 1 and 2). 


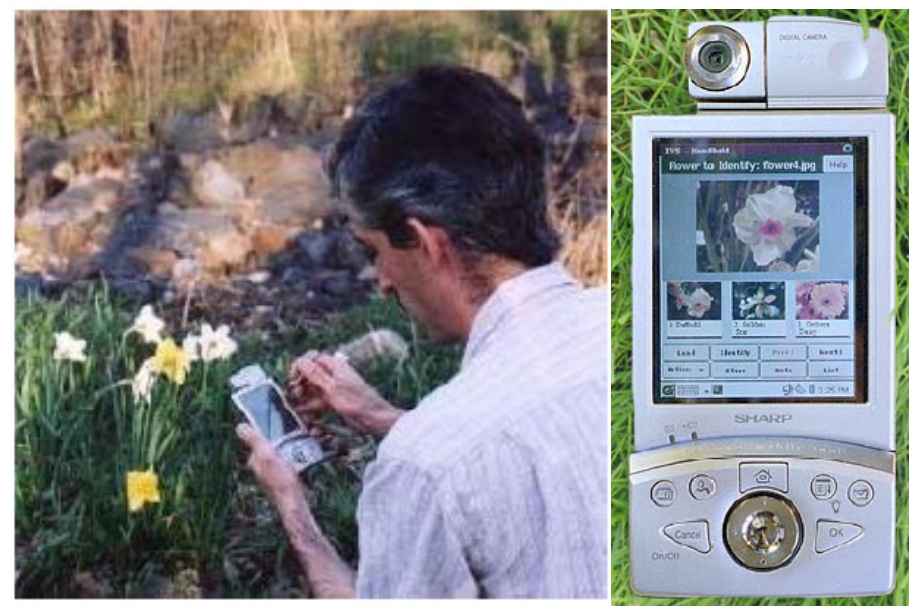

Fig. 1. IVS tool: a) in the field, b) close-up of handheld with attached camera
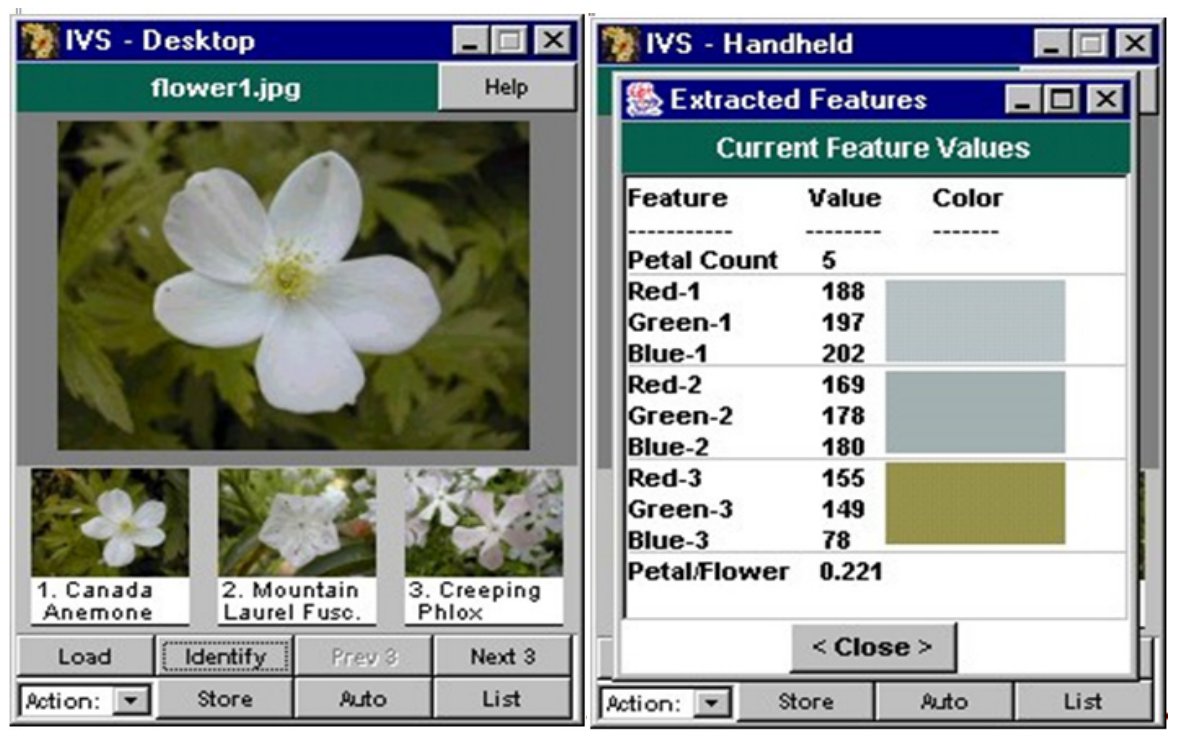

Fig. 2. IVS simulator: a) original image and top three recognition choices, b) interactive screen with feature selection

The CAVIAR model performs the usual visual pattern recognition tasks of segmentation, feature extraction, and classification. Feature extraction is performed primarily by the machine while the segmentation and classification processes are interactively performed by the human and the machine. A parametric segmentation procedure fits a "rose curve" to the flower object and the rose curve parameters can be adjusted by the human. After the machine classifier produces a list of ordered choices, the human reviews the choices to make the final decision, which is usually correct when the correct flower is in the top three choices. The current study, however, explores 
more deeply the employment of human input in the feature extraction process - for example, the degree to which items of human assistance improve system performance in terms of recognition speed and accuracy is measured.

\section{Experiment 1 - Manual, Automated, Interactive Recognition}

This experiment was conducted in three parts: human-only flower recognition (manual recognition), machine-only flower recognition (automated recognition) and human-assisted automated flower recognition (interactive recognition). Three testers (participants) were used in the three recognition tasks, and an experiment coordinator monitored the activities and recorded the results. For identification in each of the three parts of the experiment, thirty flower images, each of a different flower, were selected from a database of 535 images from 131 flower species.

\subsection{Manual Recognition}

In the manual task, the testers used a flower guidebook to assist them in the identification process. The top three flower choices and the time to identify the selected flowers were recorded. The experiment coordinator reviewed the answers provided by the participants against the answer key and identified the recognized choices as being correct or incorrect.

\subsection{Automated Recognition}

This task was performed by the IVS tool. The three testers loaded the flower images into the IVS tool and performed the automated feature extraction and recognition processes of the tool without any human input/feedback. For each flower image the application presented the top three selection choices which were recorded by the experiment coordinator. Each of the three testers performed the task separately to ensure that the same result was obtained.

\subsection{Interactive Recognition}

The interactive portion of the recognition task allows the user to provide certain feature input: petal count (the number of flower petals), petal color 1 (the most dominant color of the petal), petal color 2 (the second most dominant color of the petal), and stamen color. Additionally, the user can crop the image of the flower to eliminate possible confusion with other flowers and the background. Finally, the user can draw an outline around a petal to help the application segment a petal shape.

The interactive identification task was further divided into three subtasks, each evaluating human assistance with a different set of features. Interactive A evaluated human assistance in providing only the petal count feature ( 1 feature) with the user inputting the petal count after the application performed its automated feature extraction. Interactive B added petal colors (primary and secondary) and stamen 
color to the petal count feature (total of 4 features), and Interactive $\mathrm{C}$ added the cropped flower area (total of 5 features). The IVS process was that the user could always input or modify the machine's automated activity. Thus, the testers provided human input through the selection of feature data, using the action menu to select and input the feature data being evaluated, and then performed automated identification.

\subsection{Results of Experiment 1}

Table 1 presents a summary of the results of the experiment. Manual recognition took the longest time and provided rather low accuracy results. Automated recognition was fast but the least accurate. Although all of the human-assisted tasks yielded higher accuracy than the fully automated task, the only interactive task that performed significantly better than the human-only (manual) task was Interactive B where color recognition and petal count were performed with human assistance. Adding the cropping of the flower actually decreased recognition accuracy.

Table 1. First experiment results: 3 testers, 30 flower images

\begin{tabular}{|c|c|c|c|c|c|}
\hline \multirow{2}{*}{ Test Type } & \multicolumn{3}{|c|}{ Percent Accuracy (Top 3) } & \multirow{2}{*}{$\begin{array}{l}\text { Average } \\
\text { Accuracy }\end{array}$} & \multirow{2}{*}{$\begin{array}{c}\text { Average } \\
\text { Time } \\
\text { (sec) }\end{array}$} \\
\hline & Tester 1 & Tester 2 & Tester 3 & & \\
\hline Manual & $40.0 \%$ & $36.7 \%$ & $20.0 \%$ & $32.2 \%$ & 173 \\
\hline Automatic & $13.3 \%$ & $13.1 \%$ & $13.3 \%$ & $13.3 \%$ & 56 \\
\hline $\begin{array}{c}\text { Interactive A } \\
\text { petal count }\end{array}$ & $13.3 \%$ & $16.7 \%$ & $16.7 \%$ & $15.6 \%$ & 44 \\
\hline $\begin{array}{c}\text { Interactive B } \\
\text { plus colors }\end{array}$ & $63.3 \%$ & $60.0 \%$ & $30.0 \%$ & $51.1 \%$ & 41 \\
\hline $\begin{array}{c}\text { Interactive C } \\
\text { plus flower area }\end{array}$ & $40.0 \%$ & $36.7 \%$ & $40.0 \%$ & $38.9 \%$ & 44 \\
\hline
\end{tabular}

\section{Experiment 2 - Color Recognition}

Motivated by the results of the first experiment, a second experiment was performed to focus on human assistance in the color recognition processes (primary and secondary color of the petal and the stamen color), letting the machine perform the shape/contour recognition processes. This experiment used 20 flower images from different species and 15 testers. The results of the experiment are shown in Table 2. Recognition accuracy in the top three choices ranged from 60 to $95 \%$ with an average of 74\%, and recognition time from 43 to 63 seconds with an average of 54 seconds. This is a significant improvement in accuracy results over those in experiment 1 , while maintaining an acceptable recognition time. 
Table 2. Second experiment results: 15 testers, 20 flower images

\begin{tabular}{|c|c|c|c|c|c|c|c|c|c|c|c|c|c|c|c|}
\hline Tester & 1 & 2 & 3 & 4 & 5 & 6 & 7 & 8 & 9 & 10 & 11 & 12 & 13 & 14 & 15 \\
\hline Accuracy (Top 3) & $70 \%$ & $90 \%$ & $95 \%$ & $85 \%$ & $75 \%$ & $70 \%$ & $70 \%$ & $70 \%$ & $75 \%$ & $70 \%$ & $75 \%$ & $60 \%$ & $75 \%$ & $60 \%$ & $70 \%$ \\
\hline Avg Time (sec) & 57 & 62 & 43 & 55 & 63 & 47 & 54 & 55 & 48 & 49 & 52 & 54 & 48 & 58 & 61 \\
\hline
\end{tabular}

\section{Conclusions}

This study reveals that there is room for improvement in human-machine interaction in the feature extraction process. Human assistance in color recognition on an automated flower recognition tool showed a significant increase in accuracy while maintaining an acceptable time to accomplish the task. Assistance in other areas did not significantly increase the accuracy level, and actually decreased it when including human assistance in the shape recognition process (Interactive C). For areas where human assistance doesn't show performance increase, the approach of using automated features should not be changed. Correctly combining human and machine strengths is the approach advocated. In this study shape/contour recognition by machine combined with human input on color seems to provide the best accuracy while maintaining a reasonable time to complete the task.

Improvement of limitations is a desired goal. In this case, the human's ability in shape/contour recognition can probably not be improved. Rather, we should further explore how to improve automated capabilities in color recognition. We want to know the limit of machine capability in color only recognition.

\section{References}

1. Evans, A., Sikorski, J., Thomas, P., Cha, S.-H., Tappert, C., Zou, G., Gattani, A., Nagy, G.: Computer Assisted Visual Interactive Recognition (CAVIAR) Technology. In: 2005 IEEE International Conference on Electro-Information Technology, Lincoln, NE (May 2005) 